\title{
Long-Term Survival Outcomes of Metabolically Supported Chemotherapy with Gemcitabine- Based or FOLFIRINOX Regimen Combined with Ketogenic Diet, Hyperthermia, and Hyperbaric Oxygen Therapy in Metastatic Pancreatic Cancer: A Letter to the Editor
}

\author{
Serkan Ergözen ${ }^{a}$ Tuna Gümüşs \\ aUnderwater and Hyperbaric Medicine Department, Muğla Sıtkı Koçman University School of Medicine,

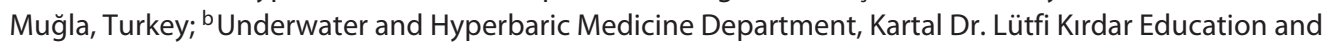 \\ Research Hospital, Istanbul, Turkey
}

\section{Dear Editor,}

When we first saw the article entitled "Long-Term Survival Outcomes of Metabolically Supported Chemotherapy with Gemcitabine-Based or FOLFIRINOX Regimen Combined with Ketogenic Diet, Hyperthermia, and Hyperbaric Oxygen Therapy in Metastatic Pancreatic Cancer" by Mehmet Salih Iyikesici [1], we were very excited as specialists on underwater and hyperbaric medicine. However, when we have finished reading the article, unfortunately many very serious concerns popped up in our minds.

Firstly, we wanted to learn more about the hyperbaric chamber mentioned in the article (Quamvis ${ }^{320}{ }^{\circledR}$ hyperbaric chamber; OxyHealth) and made an internet search. We found the specifications of the chamber on the producer's website. We found out that the operating pressure of the chamber was only 1.3 ATA [2], but the author declares that 1.5 ATA was used [1]. Technically, these chambers have a safety valve in order not to exceed this safe operating pressure declared by the producer. So, how these 1.5 ATA could be reached must be clarified by the author. It would be a serious concern in terms of safety if this chamber was used above the operating pressure.

Secondly, the author must clarify whether he used pure (100\%) oxygen or air during the treatment, otherwise it seems to us that this application is only hyper-

baric air, although the author says it is hyperbaric oxygen therapy (HBOT). If pure oxygen was used, the author must also mention which method/methods he used for delivering oxygen to the patients (e.g., mask, hood, or free oxygen flow into the chamber). This is a very important issue because if someone uses a hyperbaric chamber for HBOT, the chamber has to have an oxygen analyzer for fire safety; this is also a legal policy in our country (Turkey) [3]. The mentioned chamber does not have an oxygen sensor in its specifications [2].

Thirdly, cancer treatment is not an approved indication of HBOT in Turkey, Europe, or the USA, neither alone nor as an adjuvant treatment [3-6]. Thus, this study must have been designed as a prospective study by receiving approval from an ethics committee for clinical trials, not as a retrospective study without any ethics approval.

Fourthly, the interaction between HBOT and chemotherapeutics is not clear, especially regarding timing. However, the author used HBOT after each metabolically supported chemotherapy protocol, both regimes including cis-platinum and some other chemotherapeutics. Especially cis-platinum use concurrently with HBOT is accepted as a contraindication $[7,8]$. This means that the methodology of this study is also questionable.

Fifthly, the criteria for selecting patients for HBOT are not mentioned in the article. This point is very im- 
portant for patients' safety in terms of complications of HBOT. As far as we understood from the paper, some patients had lung metastasis and this condition was to be evaluated by an underwater and hyperbaric medicine specialist before the treatment, but we are not sure whether the author had any consultation for patient selection.

Lastly, legal regulations in Turkey authorize only underwater and hyperbaric medicine specialists to apply HBOT, and also the institution must get permission from the Turkish Ministry of Health to establish a hyperbaric oxygen treatment center. It seems to us that HBOT in this study might have been applied in an unauthorized institution and also by an unauthorized person. This is another very serious concern.

We hope the author is going to clarify our concerns about his article.

\section{Acknowledgment}

The authors thank Prof. Dr. Eda Üstünel for language editing.

\section{Conflict of Interest Statement}

The authors have no conflicts of interest to declare.

\section{Funding Sources}

There was no funding.

\section{Author Contributions}

The authors declare that they prepared this letter to the editor together.

\section{References}

1 Iyikesici MS. Long-term survival outcomes of metabolically supported chemotherapy with gemcitabine-based or FOLFIRINOX regimen combined with ketogenic diet, hyperthermia, and hyperbaric oxygen therapy in metastatic pancreatic cancer. Complement Med Res. 2020;27(1):31-9.

2 OxyHealth. Quamvis $320^{\circledR}$ hyperbaric chamber. Simple for patients to handle. Available from: https://www.oxyhealth.com/quamvis320.html.
3 Available from: https://www.mevzuat.gov.tr/ mevzuat? MevzuatNo $=4809 \&$ MevzuatTur $=7$ \&MevzuatTertip $=5$.

4 Available from: https://www.mevzuat.gov.tr/ mevzuat?MevzuatNo=17229\&MevzuatTur= 9\&MevzuatTertip $=5$.

5 Mathieu D, Marroni A, Kot J. Tenth European Consensus Conference on Hyperbaric Medicine: recommendations for accepted and non-accepted clinical indications and practice of hyperbaric oxygen treatment. Diving Hyperb Med. 2017 Mar;47(1):24-32.
6 Undersea \& Hyperbaric Medical Society. In dications for hyperbaric oxygen therapy. Available from: https://www.uhms.org/resources/hbo-indications.html.

7 Baude J, Cooper JS. Hyperbaric contraindicated chemotherapeutic agents. In: StatPearls [Internet]. Treasure Island, FL: StatPearls Publishing; 2020. Available from: https:// www.ncbi.nlm.nih.gov/books/NBK560873/.

8 Ergözen S, Kaya E. Hyperbaric oxygen treatment. Med J Mugla Sitki Kocman Univ. 2018; 5(3):46-50. 